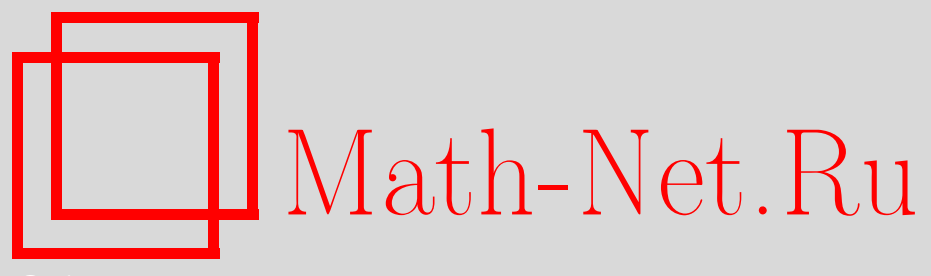

А. В. Мартыненко, А. Ф. Тедеев, В. Н. Шраменко, Задача Коши для вырождающегося параболического уравнения с неоднородной плотностью и источником в классе медленно стремящихся к нулю начальных функций, Изв. РАН. Сер. матем., 2012, том 76, выпуск 3, 139-156

DOI: https://doi.org/10.4213/im6847

Использование Общероссийского математического портала Math-Net.Ru подразумевает, что вы прочитали и согласны с пользовательским соглашением http://www . mathnet.ru/rus/agreement

Параметры загрузки:

IP: 3.82 .47 .9

26 апреля 2023 г., 04:06:57

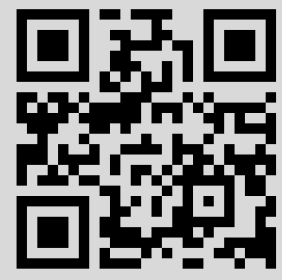


УДК 517.946

\author{
А. В. Мартыненко, А. Ф. Тедеев, В. Н. Шраменко
}

\title{
Задача Коши \\ для вырождающегося параболического уравнения \\ с неоднородной плотностью и источником в классе медленно стремящихся к нулю начальных функций
}

Для вырождающегося параболического уравнения с источником и неоднородной плотностью вида $\rho(x) u_{t}=\operatorname{div}\left(u^{m-1}|D u|^{\lambda-1} D u\right)+\rho(x) u^{p}$ рассматривается задача Коши с начальной функцией, медленно стремящейся к нулю при $|x| \rightarrow \infty$. Найдены условия существования и несуществования решения задачи Коши глобально по времени, которые в значительной мере зависят от поведения начальной функции при $|x| \rightarrow \infty$. В случае глобальной разрешимости получена точная оценка решения при больших значениях времени.

Библиография: 28 наименований.

Ключевые слова: неоднородная плотность, вырождающееся параболическое уравнение, режим с обострением, медленно убывающая начальная функция.

\section{§ 1. Введение}

Рассмотрим следующую задачу Коши:

$$
\begin{gathered}
\rho(x) u_{t}=\operatorname{div}\left(u^{m-1}|D u|^{\lambda-1} D u\right)+\rho(x) u^{p}, \\
(x, t) \in Q_{T}=\mathbb{R}^{N} \times(0, T), \quad T>0, \quad N \geqslant 1, \\
u(x, 0)=u_{0}(x), \quad x \in \mathbb{R}^{N} .
\end{gathered}
$$

Задача (1), (2) при различных значениях параметров $m, \lambda$ и $\rho(x)$ изучалась многими авторами. В работах [1], [2] было впервые установлено, что даже в простейшем случае $(m=1, \lambda=1$ и $\rho(x) \equiv 1)$ уравнение (1) обладает решениями, которые становятся неограниченными за конечное время (“взрываются"), т. е. задача (1), (2) может не иметь глобальных по времени решений. Этот результат дал мощный импульс целому ряду исследований, посвященных получению условий существования и несуществования в целом по времени решений уравнения с источником вида (1).

В случае однородной плотности $(\rho(x) \equiv 1)$ такие условия для уравнения пористой среды $(\lambda=1, m>1)$ были получены в [3]-[5], для уравнения неньютоновской фильтрации $(\lambda>1, m=1)-$ в [6], [7], а для уравнения с двойной нелинейностью $(\lambda>1, m>1)$ - в [8], [9]. Было установлено, что существует критическое значение $p^{*}$ показателя $p$ такое, что при $1<p<p^{*}$ любое нетривиальное решение "взрывается", а при $p>p^{*}$ и “достаточно малой" начальной 
функции решение существует глобально по времени. Результаты такого вида принято называть теоремами типа Фуджить, а $p^{*}$ - критическим показателем Фуджиты. В частности, из результатов работы [9] следует, что для задачи $(1),(2)$ при $\rho(x) \equiv 1$ показатель Фуджиты имеет вид $p^{*}=m+\lambda-1+(\lambda+1) / N$, а условие "достаточной малости" начальной функции заключается в том, что

$$
\left\|u_{0}\right\|_{L^{1}\left(\mathbb{R}^{N}\right)}+\left\|u_{0}\right\|_{L^{q}\left(\mathbb{R}^{N}\right)}<\delta
$$

при некотором $q>N(p-m-\lambda+1) /(\lambda+1)$ и достаточно малом $\delta$. Подробное изложение основных результатов для случая $\rho(x) \equiv 1$ можно найти в монографии [4] и обзорных статьях [10], [11].

Задача $(1),(2)$ при $\rho(x)=(1+|x|)^{-l}, l \geqslant 0$, была рассмотрена в [12]. Оказалось, что свойства решения существенно зависят от значения $l$. Если $l<\lambda+1<N$, то $p^{*}=m+\lambda-1+(\lambda+1-l) /(N-l)$, а условие "достаточной малости" начальной функции аналогично условию (3) и имеет вид $\left\|u_{0}\right\|_{L_{\rho}^{1}\left(\mathbb{R}^{N}\right)}+\left\|u_{0}\right\|_{L_{\rho}^{q}\left(\mathbb{R}^{N}\right)}<\delta$ при некотором $q>(N-l)(p-m-\lambda+1) /(\lambda+1-l)$ и достаточно малом $\delta$. Если $\min \{l, N\}>\lambda+1$, то $p^{*}=m+\lambda-1$ (т. е. показатель Фуджиты не зависит от $l$ ) и для глобальной разрешимости достаточно того, чтобы $\left\|u_{0}\right\|_{L_{\rho}^{1}\left(\mathbb{R}^{N}\right)}<\infty,\left\|u_{0}\right\|_{L_{\rho}^{q}\left(\mathbb{R}^{N}\right)}<\delta$. Наконец, в случае $N \leqslant l \leqslant \lambda+1$ любое нетривиальное решение "взрывается" при $p>m+\lambda-1$.

Отметим, что изменение качественных свойств решения при увеличении $l$, полученное в [12], характерно для уравнений с неоднородной плотностью. Например, для уравнения (1) без источника в случае медленной диффузии $(m+\lambda-2>0)$ нарушаются некоторые свойства вырождающихся параболических уравнений, такие как стабилизация к нулю решения при $t \rightarrow \infty$ и конечная скорость распространения носителя, если $\rho(x)$ достаточно быстро убывает на бесконечности (см. [13]-[21]).

Заметим, что изложенные выше результаты по условиям глобальной разрешимости были получены в предположении $u_{0}(x) \in L_{\rho}^{1}\left(\mathbb{R}^{N}\right)$. Если начальная функция интегрируема лишь локально, то, как показано в [22], значение показателя $p^{*}$ зависит от поведения начальной функции при $|x| \rightarrow \infty$. В работе [22] рассматривалась задача $(1),(2)$ при $\rho(x) \equiv 1, \lambda=1, p>m>1, u_{0}(x) \sim|x|^{-a}$ при больших $x, 0 \leqslant a<N$. Для такой начальной функции $p^{*}=m+2 / a$. Аналогичный результат был получен в [23] для задачи $(1),(2)$ при $\rho(x) \equiv 1$, $m+\lambda-2<0$ (быстрая диффузия).

Основной целью настоящей работы является нахождение условий существования и несуществования в целом по времени решений задачи (1), (2) в классе начальных функций, вообще говоря, не принадлежащих $L_{\rho}^{1}\left(\mathbb{R}^{N}\right)$. В случае существования решения в целом по времени мы получаем оценку решения, которая является точной при больших значениях $t$.

Всюду далее предполагаем, что $1<\lambda+1<N, m+\lambda-2>0, p>m+\lambda-1$, $\rho(x)=|x|^{-l}, 0 \leqslant l<\lambda+1$ и $u_{0}(x)-$ неотрицательная измеримая функция из $L_{\rho, \mathrm{loc}}^{1}\left(\mathbb{R}^{N}\right)$. Также предположим, что для некоторого $\alpha \in(0, N-l)$ и $\theta=$ $\max \{1, l(N-\lambda-1) /(\alpha(\lambda+1))\}$

$$
\left.\left\|\left|u_{0}\right|\right\|\right|_{\rho, \theta}=\sup _{x_{0} \in \mathbb{R}^{N}}\left(1+\left|x_{0}\right|\right)^{\alpha}\left(f_{B_{d\left(\left|x_{0}\right|\right)}\left(x_{0}\right)} \rho u_{0}^{\theta}(x) d x\right)^{\frac{1}{\theta}}<\infty,
$$


где $f_{E} \rho v(x) d x=\int_{E} \rho v(x) d x / \int_{E} \rho d x, B_{R}\left(x_{0}\right)=\left\{x \in \mathbb{R}^{N}:\left|x-x_{0}\right| \leqslant R\right\}$ и $d(a)=(1+a)^{(\alpha \theta+l) / N}, a \geqslant 0$. Норма $\||\cdot|\|_{\rho, \theta}$ при $\rho(x) \equiv 1$ совпадает с нормой ||$|\cdot| \|_{\theta}$, которая была введена и подробно изучена в работе [5]. Многие свойства нормы ||$|\cdot|||_{\theta}$ без труда переносятся на норму $\left.\||\cdot|\|\right|_{\rho, \theta}$ при $\rho(x)=|x|^{-l}$.

Условие (4) характеризует поведение функции при $x \rightarrow \infty$. Примером начальной функции, удовлетворяющей этому условию, служит $u_{0}(x) \backsim|x|^{-\alpha}$ при больших $x, 0<\alpha<N-l$.

Будем говорить, что $u(x, t)$ - обобщенное решение (или просто решение) задачи (1), (2) в $Q_{T}=\mathbb{R}^{N} \times(0, T)$, если

$$
\begin{gathered}
u \in L_{\mathrm{loc}}^{\infty}\left(Q_{T}\right) \cap C\left((0, T), L_{\rho, \text { loc }}^{2}\left(\mathbb{R}^{N}\right)\right), \\
u^{m-1}|D u|^{\lambda+1} \in L_{\mathrm{loc}}^{1}\left(Q_{T}\right), \quad u \in L_{\rho, \text { loc }}^{p}\left(Q_{T}\right), \\
u(x, t) \rightarrow u_{0}(x) \quad \text { при } \quad t \rightarrow 0 \quad \text { в } \quad L_{\rho, \text { loc }}^{1}\left(\mathbb{R}^{N}\right)
\end{gathered}
$$

и выполнено интегральное тождество

$$
\int_{0}^{T} \int_{\mathbb{R}^{N}}\left\{-\rho u \varphi_{t}+u^{m-1}|D u|^{\lambda-1} D u D \varphi\right\} d x d t=\int_{0}^{T} \int_{\mathbb{R}^{N}} \rho u^{p} \varphi d x d t
$$

для произвольной пробной функции $\varphi(x, t) \in C_{0}^{1}\left(Q_{T}\right)$.

ЗАмЕчАниЕ 1. Если не оговорено противное, то всюду далее через $\gamma, \gamma_{1}$, $\gamma_{2}, \ldots$ будем обозначать постоянные, которые зависят только от параметров задачи $l, m, \lambda, p, N, \alpha$.

Основные результаты настоящей статьи могут быть сформулированы следующим образом.

TEOPEMA 1. Пусть $p>p_{\alpha}^{*}(l)=m+\lambda-1+(\lambda+1-l) / \alpha u$

$$
\left\|u_{0} \mid\right\|_{\rho, \theta}+\left\|u_{0}\right\|_{L_{\rho}^{q}\left(\mathbb{R}^{N}\right)} \leqslant \delta
$$

где $q>Q=(N-l)(p-m-\lambda+1) /(\lambda+1-l)$ и $\delta>0-$ достаточно малое число, зависящее только от параметров задачи (1), (2).Тогда задача (1), (2) имеет глобальное по времени решение и для любого $t \in(0, \infty)$ справедлива оценка

$$
\|u(\cdot, t)\|_{\infty, \mathbb{R}^{N}} \leqslant \gamma_{1} t^{-\frac{N-l}{H_{l, \theta}}}\left(\gamma_{2}+\left(t\left|\left\|u_{0} \mid\right\|_{\rho, \theta}^{m+\lambda-2}\right)^{\frac{N-\alpha \theta-l}{G_{\alpha}}}\right)^{\frac{\lambda+1-l}{H_{l, \theta}}}\left\|\left|u_{0} \|\right|_{\rho, \theta}^{\frac{(\lambda+1-l) \theta}{H_{l, \theta}}},\right.\right.
$$

где

$$
H_{l, \omega}=(N-l)(m+\lambda-2)+(\lambda+1-l) \omega, \quad G_{\alpha}=\alpha(m+\lambda-2)+\lambda+1-l .
$$

ЗАмЕчАнИЕ 2. Очевидно, что при $t<C\left|\left\|u_{0} \mid\right\|_{\rho, \theta}^{-(m+\lambda-2)}\right.$ оценка (6) имеет вид

$$
\|u(\cdot, t)\|_{\infty, \mathbb{R}^{N}} \leqslant \gamma t^{-\frac{N-l}{H_{l, \theta}}}\left\|\left|u_{0}\right|\right\|_{\rho, \theta}^{\frac{(\lambda+1-l) \theta}{H_{l, \theta}}},
$$

а при $t \geqslant\left. C||\left|u_{0}\right|\right|_{\rho, \theta} ^{-(m+\lambda-2)}$ оценка (6) имеет вид

$$
\|u(\cdot, t)\|_{\infty, \mathbb{R}^{N}} \leqslant \gamma t^{-\frac{\alpha}{G_{\alpha}}}\left|\left\|u_{0} \mid\right\|_{\rho, \theta}^{\frac{\lambda+1-l}{G_{\alpha}}} .\right.
$$


ТЕОрема 2. Пусть $p<p_{\alpha}^{*}(l), u(x, t)$ - решение задачи (1), (2) и начальная функиия $u_{0}(x)$ удовлетворяет условию

$$
\left(R+\left|x_{0}\right|\right)^{\alpha(1-\theta)} f_{B_{R}\left(x_{0}\right)} \rho u_{0}^{1-\theta}(x) d x \geqslant \gamma_{1}
$$

при некоторых $x_{0} \in \mathbb{R}^{N}, \theta \in(0,1)$ и произвольном $R>0$. Тогда $u(x, t)$ "взрьвается" за конечное время, т.е. найдутся такие $0<R_{1}<\infty u 0<T<\infty$, ymo

$$
\int_{B_{R_{1}}\left(x_{0}\right)} \rho u^{1-\theta}(x, t) d x \rightarrow \infty \quad \text { npu } \quad t \rightarrow T .
$$

ЗАмЕчАНИЕ 3. Поскольку при доказательстве основных результатов будут использоваться локальные энергетические оценки, то модельность уравнения (1) не принципиальна.

ЗАмЕчАНИЕ 4. При доказательстве основных результатов мы использовали подходы из работ [5], [12], [20], [24] и [25].

\section{§ 2. Вспомогательные утверждения}

Пусть $w(x) \in L_{\rho, \text { loc }}^{1}\left(\mathbb{R}^{N}\right)$; введем в рассмотрение норму

$$
[w]_{\theta}=\sup _{x_{0} \in \mathbb{R}^{N}} \sup _{R>r_{\theta}\left(\left|x_{0}\right|\right)}\left(R+\left|x_{0}\right|\right)^{\alpha}\left(f_{B_{R}\left(x_{0}\right)} \rho|w(x)|^{\theta} d x\right)^{\frac{1}{\theta}},
$$

где $\theta, \alpha$ такие же, как в определении нормы ||$|\cdot|||_{\rho, \theta}$ и $r_{\theta}(a)-$ функция, заданная неявно уравнением

$$
\frac{r_{\theta}^{N}}{\left(r_{\theta}+a\right)^{\alpha \theta+l}}=1
$$

Очевидно, что функция $r_{\theta}(a)$ определена и непрерывна при $a \geqslant 0$, кроме того, $r_{\theta}(a)$ строго возрастает и $r_{\theta}(0)=1$.

Нашей ближайшей целью является доказательство эквивалентности норм $\||| \cdot||_{\rho, \theta}$ и $[\cdot]_{\theta}$, для чего понадобятся следующие леммы.

Лемма 1. Пусть $\mu<N, s>0$; тогда найдутся положительные постояннье $C_{1}(N, \mu), C_{2}(N, \mu), C_{3}(N, \mu, s)$ и $C_{4}(N, \mu, s)$ такие, что для любых $R>0$, $x_{0} \in \mathbb{R}^{N}$

$$
\begin{aligned}
& C_{1} R^{N}\left(R+\left|x_{0}\right|\right)^{-\mu} \leqslant \int_{B_{R}\left(x_{0}\right)}|x|^{-\mu} d x \leqslant C_{2} R^{N}\left(R+\left|x_{0}\right|\right)^{-\mu}, \\
& C_{3} \int_{B_{R}\left(x_{0}\right)}|x|^{-\mu} d x \leqslant \int_{B_{s R}\left(x_{0}\right)}|x|^{-\mu} d x \leqslant C_{4} \int_{B_{R}\left(x_{0}\right)}|x|^{-\mu} d x .
\end{aligned}
$$

ДокАзАтельство. Неравенство (9) следует из того, что при $\left|x_{0}\right| \geqslant 3 R$

$$
\frac{1}{3}\left(\left|x_{0}\right|+R\right) \leqslant\left|x_{0}\right|-R \leqslant|| x-x_{0}|-| x_{0}|| \leqslant|x| \leqslant\left|x_{0}\right|+R,
$$


а при $\left|x_{0}\right|<3 R$

$$
\int_{B_{R}\left(x_{0}\right)}|x|^{-\mu} d x \leqslant \int_{B_{4 R}(0)}|x|^{-\mu} d x .
$$

Неравенство (10) является очевидным следствием (9). Лемма доказана.

Лемма 2. Пусть $а \geqslant 0 ;$ тогда найдется положительная постоянная $C_{5}(N, \alpha, \theta, l)$ такал, что

$$
d(a) \leqslant r_{\theta}(a) \leqslant C_{5} d(a) .
$$

ДокАЗАТЕЛЬСтво. Поскольку $r_{\theta}(a) \geqslant 1$, то $\left(r_{\theta}(a)\right)^{N} /(1+a)^{\alpha \theta+l} \geqslant 1$, следовательно, первое из неравенств (11) выполнено.

Второе неравенство в $(11)$ следует из того, что $r_{\theta}(a) \leqslant 2^{(\alpha \theta+l) /(N-\alpha \theta-l)}$ при $r_{\theta}(a) \geqslant a$ и $r_{\theta}(a) \leqslant(2 a)^{(\alpha \theta+l) / N}$ при $r_{\theta}(a)<a$. Лемма доказана.

Под минимальной $\varepsilon$-сетью множества $F \subset \mathbb{R}^{N}$ будем понимать множество $E_{\varepsilon}(F)$ такое, что $E_{\varepsilon}(F) \subset F ;|x-y| \geqslant \varepsilon$ для любых $x, y \in E_{\varepsilon}(F)$ при $x \neq y$; для любого $x \in F$ существует $y \in E_{\varepsilon}(F)$ такое, что $|x-y| \leqslant \varepsilon$. Хорошо известно, что минимальная $\varepsilon$-сеть существует для любого множества $F \subset \mathbb{R}^{N}$, кроме того, будет справедлива следующая лемма.

Лемма 3. Пусть $F$ - произвольное множество в $\mathbb{R}^{N} ;$ тогда:

1) $F \subset \bigcup_{x \in E_{\varepsilon}(F)} B_{\varepsilon}(x)$;

2) любая точка множества $F$ принадлежит не более чем $5^{N}$ шарам вида $B_{2 \varepsilon}(x)$, где $x \in E_{\varepsilon}(F)$.

ДоказАтельство. Утверждение 1) тривиально. Докажем утверждение 2). Пусть $y \in F$ и $x_{1}, x_{2}, \ldots, x_{k} \in B_{2 \varepsilon}(y) \cap E_{\varepsilon}(F)$. Тогда $\bigcup_{i=1}^{k} B_{\varepsilon / 2}\left(x_{i}\right) \subset B_{5 \varepsilon / 2}(y)$, причем $B_{\varepsilon / 2}\left(x_{i}\right) \cap B_{\varepsilon / 2}\left(x_{j}\right)=\varnothing$ при $i \neq j$, следовательно,

$$
\sum_{i=1}^{k}\left|B_{\varepsilon / 2}\left(x_{i}\right)\right|=\left|\bigcup_{i=1}^{k} B_{\varepsilon / 2}\left(x_{i}\right)\right| \leqslant\left|B_{5 \varepsilon / 2}(y)\right| .
$$

Таким образом, $k \omega_{N}(\varepsilon / 2)^{N} \leqslant \omega_{N}(5 \varepsilon / 2)^{N}$, где $\omega_{N}$ - мера единичного шара в $\mathbb{R}^{N}$. Отсюда следует, что $k \leqslant 5^{N}$, а поскольку $y \notin B_{2 \varepsilon}(x)$ при $x \notin B_{2 \varepsilon}(y)$, то утверждение 2) верно.

ЛЕмМА 4. Пусть

$$
\begin{gathered}
S_{0}=B_{1}(0), \quad S_{k}=B_{2^{k}}(0) \backslash B_{2^{k-1}}(0), \quad k=1,2, \ldots, \\
\widetilde{S}_{k}=\bigcup_{x \in S_{k}} B_{d\left(2^{k}\right)}(x), \quad k=0,1,2, \ldots .
\end{gathered}
$$

Тогда любая точка пространства $\mathbb{R}^{N}$ лежит в не более чем $\widetilde{n}=\lfloor(2 N+\alpha \theta+$

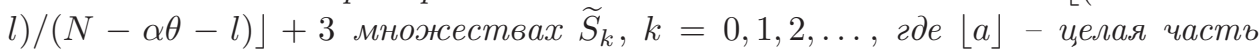
вещественного числа а.

ДокАЗАТЕЛЬСтво. Легко видеть, что при $k=0,1,2, \ldots$ множество $\widetilde{S}_{k}$ представимо в виде

$$
\widetilde{S}_{k}=\left\{x \in \mathbb{R}^{N}: 2^{k-1}-d\left(2^{k}\right) \leqslant|x| \leqslant 2^{k}+d\left(2^{k}\right)\right\} .
$$


Из условий, налагаемых на параметры $\alpha, l$ и $N$, следует $(\alpha \theta+l) / N<1$, поэтому при $k=0,1,2, \ldots$

$$
2^{k}+d\left(2^{k}\right) \leqslant 2^{k}+\left(2^{k+1}\right)^{\frac{\alpha \theta+l}{N}} \leqslant 2^{k+2} .
$$

Далее рассмотрим два случая:

1) $k \geqslant(2 N+\alpha \theta+l) /(N-\alpha \theta-l)$;

2) $k<(2 N+\alpha \theta+l) /(N-\alpha \theta-l)$.

В случае 1) имеем

$$
2^{k-1}-d\left(2^{k}\right) \geqslant 2^{k-1}-\left(2^{k+1}\right)^{\frac{\alpha \theta+l}{N}} \geqslant 2^{k-2},
$$

что вместе с (12) дает

$$
\widetilde{S}_{k} \subset \bigcup_{i=1}^{5} S_{k-3+i}
$$

В случае 2) получаем

$$
\widetilde{S}_{k} \subset \bigcup_{i=1}^{\widetilde{n}} S_{i} .
$$

Поскольку $(2 N+\alpha \theta+l) /(N-\alpha \theta-l) \geqslant 2$, при $k=0,1,2, \ldots$ множество $\widetilde{S}_{k}$ лежит в объединении не более чем $\widetilde{n}$ множеств $S_{k}, k=0,1,2, \ldots$. Отсюда следует справедливость леммы.

ТеОрема 3. Пусть $w(x)$ - измеримая функиия из $L_{\rho, \mathrm{loc}}^{1}\left(\mathbb{R}^{N}\right)$, и пусть $\||w|\|_{\rho, \theta}<\infty$. Тогда найдутся положительные постоянные $C_{6}(N, \alpha, \theta, l)$, $C_{7}(N, \alpha, \theta, l)$ такие, что

$$
C_{6}|||w|\left\|_{\rho, \theta} \leqslant[w]_{\theta} \leqslant C_{7} \mid\right\| w \|_{\rho, \theta} .
$$

ДокаЗАтельство. Из лемм 1,2 следует, что $B_{d\left(\left|x_{0}\right|\right)}\left(x_{0}\right) \subset B_{r_{\theta}\left(\left|x_{0}\right|\right)}\left(x_{0}\right)$ и существуют положительные постоянные $C_{8}(N, \alpha, \theta, l), C_{9}(N, \alpha, \theta, l)$ такие, что

$$
\begin{gathered}
\left(1+\left|x_{0}\right|\right) \leqslant C_{8}\left(r_{\theta}\left(\left|x_{0}\right|\right)+\left|x_{0}\right|\right) \\
\int_{B_{r_{\theta}\left(\left|x_{0}\right|\right)}\left(x_{0}\right)} \rho(x) d x \leqslant C_{9} \int_{B_{d\left(\left|x_{0}\right|\right)}\left(x_{0}\right)} \rho(x) d x .
\end{gathered}
$$

Используя последние неравенства, получаем

$$
\begin{aligned}
& \||w|\|_{\rho, \theta} \leqslant \sup _{x_{0} \in \mathbb{R}^{N}} \frac{\left(1+\left|x_{0}\right|\right)^{\alpha}}{\left(r_{\theta}\left(\left|x_{0}\right|\right)+\left|x_{0}\right|\right)^{\alpha}}\left(\frac{\int_{B_{r_{\theta}\left(\left|x_{0}\right|\right)}\left(x_{0}\right)} \rho(x) d x}{\int_{B_{d\left(\left|x_{0}\right|\right)}\left(x_{0}\right)} \rho(x) d x}\right)^{\frac{1}{\theta}} \\
& \times\left(f_{B_{r_{\theta}\left(\left|x_{0}\right|\right)}\left(x_{0}\right)} \rho|w(x)|^{\theta} d x\right)^{\frac{1}{\theta}} \leqslant C_{8}^{\alpha} C_{9}^{\frac{1}{\theta}}[w]_{\theta} .
\end{aligned}
$$

Таким образом, первое из неравенств (13) доказано, докажем второе.

Пусть $x_{0} \in \mathbb{R}^{N}, R \geqslant r_{\theta}\left(\left|x_{0}\right|\right)$ и $n$ - натуральное число такое, что $B_{R}\left(x_{0}\right) \subset$ $B_{2^{n}}\left(x_{0}\right)$. Рассмотрим множества $M_{k}=B_{R}\left(x_{0}\right) \cap S_{k}$, где $S_{k}$ - множества из условия леммы $4, k=\overline{0, n}$. Поскольку множество $M_{k}$ предкомпактно при 
$k=\overline{0, n}$, то существует конечная минимальная $d\left(2^{k-1}\right)$-сеть $E_{d\left(2^{k-1}\right)}\left(M_{k}\right)=$ $\left\{x_{1}^{(k)}, x_{2}^{(k)}, \ldots, x_{m_{k}}^{(k)}\right\}$. Отсюда следует, что шары $B_{d\left(2^{k-1}\right)}\left(x_{i}^{(k)}\right), k=\overline{0, n}, i=$ $\overline{1, m_{k}}$ образуют покрытие шара $B_{R}\left(x_{0}\right)$. Так как $M_{k} \subset S_{k}$, то при $k=\overline{0, n}$ и $i=\overline{1, m_{k}}$

$$
2^{k-1} \leqslant\left|x_{i}^{(k)}\right| \leqslant 2^{k},
$$

поэтому шары $B_{i, k}=B_{d\left(\left|x_{i}^{(k)}\right|\right)}\left(x_{i}^{(k)}\right), k=\overline{0, n}, i=\overline{1, m_{k}}$, также образуют покрытие шара $B_{R}\left(x_{0}\right)$. Тогда, используя лемму 1 и очевидное соотношение

$$
2(1+a) \geqslant d(a)+a,
$$

получаем

$$
\begin{gathered}
\left(R+\left|x_{0}\right|\right)^{\alpha} f_{B_{R}\left(x_{0}\right)} \rho|w(x)|^{\theta} d x \leqslant \sup _{x_{0} \in \mathbb{R}^{N}} \frac{\left(R+\left|x_{0}\right|\right)^{\alpha \theta}}{\int_{B_{R}\left(x_{0}\right)} \rho(x) d x} \\
\times \sum_{k=1}^{n} \sum_{i=1}^{m_{k}} \frac{\int_{B_{i, k}} \rho(x) d x}{\left(1+\left|x_{i}^{(k)}\right|\right)^{\alpha \theta}}\left(\left(1+\left|x_{i}^{(k)}\right|\right)^{\alpha \theta} f_{B_{i, k}} \rho|w(x)|^{\theta} d x\right) \\
\leqslant\left.\frac{C_{10}(N, \alpha, \theta, l)}{\int_{B_{R}\left(x_{0}\right)}|x|^{-l-\alpha \theta} d x} \sum_{k=1}^{n} \sum_{i=1}^{m_{k}} \int_{B_{i, k}}|x|^{-l-\alpha \theta} d x|||w|\right|_{\rho, \theta} ^{\theta} .
\end{gathered}
$$

Для завершения доказательства необходимо оценить

$$
\sum_{k=1}^{n} \sum_{i=1}^{m_{k}} \int_{B_{i, k}\left(x_{0}\right)}|x|^{-l-\alpha \theta} d x .
$$

Для этого заметим, что согласно (14) при $k=\overline{0, n}, i=\overline{1, m_{k}}$

$$
B_{i, k} \subset B_{d\left(2^{k}\right)}\left(x_{i}^{(k)}\right),
$$

а из лемм 3,4 следует, что любая точка из $\mathbb{R}^{N}$ лежит в не более чем $5^{N} \widetilde{n}$ шарах $B_{d\left(2^{k}\right)}\left(x_{i}^{(k)}\right), k=\overline{0, n}, i=\overline{1, m_{k}}$, тем более, это верно для шаров $B_{i, k}$. С другой стороны, из того, что $x_{i}^{(k)} \leqslant\left|x_{0}\right|+R$ и $R \geqslant r_{\theta}\left(\left|x_{0}\right|\right) \geqslant d\left(\left|x_{0}\right|\right)$, следует

$$
d\left(\left|x_{i}^{(k)}\right|\right) \leqslant\left(1+\left|x_{0}\right|+R\right)^{\frac{\alpha \theta+l}{N}} \leqslant\left(R^{\frac{N}{\alpha \theta+l}}+R\right)^{\frac{\alpha \theta+l}{N}} \leqslant 2 R,
$$

поэтому

$$
\bigcup_{k=0}^{n} \bigcup_{i=1}^{m_{k}} B_{i, k} \subset B_{3 R}\left(x_{0}\right) .
$$

Из неравенства (10) и включений (16), (17) следует, что

$$
\begin{aligned}
\sum_{k=1}^{n} \sum_{i=1}^{m_{k}} \int_{B_{i, k}}|x|^{-l-\alpha \theta} d x & \leqslant 5^{N} \tilde{n} \int_{B_{3 R}\left(x_{0}\right)}|x|^{-l-\alpha \theta} d x \\
& \leqslant C_{11}(N, \alpha, \theta, l) \int_{B_{R}\left(x_{0}\right)}|x|^{-l-\alpha \theta} d x .
\end{aligned}
$$

Из оценок (15) и (18) следует справедливость второго из неравенств (13). Теорема доказана. 
Норма $[\cdot]_{\theta}$, будучи эквивалентной норме ||$|\cdot| \|_{\rho, \theta}$, лучше соответствует структуре уравнения (1) и с технической точки зрения более удобна для описания начальной функции. Доказательство теоремы 1 будет проведено для нормы $[\cdot]_{\theta}$.

\section{§ 3. Доказательство теоремы 1}

Пусть $B_{j}=\left\{x \in \mathbb{R}^{N}:|x| \leqslant j\right\}, j \in \mathbb{N}$, и пусть $\left\{u_{0 j}\right\}_{j=1}^{\infty}-$ последовательность функций таких, что $u_{0 j} \in C_{0}^{\infty}\left(B_{j}\right), u_{0 j} \rightarrow u_{0}$ в $L_{\rho, \text { loc }}^{1}\left(\mathbb{R}^{N}\right) \cap L_{\rho}^{q}\left(\mathbb{R}^{N}\right)$ и $\left[u_{0 j}-u_{0}\right]_{\theta} \rightarrow 0$ при $n \rightarrow \infty$.

Аппроксимируем задачу (1), (2) с помощью начально-краевых задач

$$
\begin{gathered}
\rho \frac{\partial u_{j}}{\partial t}=\operatorname{div}\left(u_{j}^{m-1}\left|D u_{j}\right|^{\lambda-1} D u_{j}\right)+\rho u_{j} \min \left\{j, u_{j}^{p-1}\right\} \\
u_{j}(x, t)=0, \quad x \in \partial B_{j} \\
u_{j}(x, 0)=u_{0, j}(x) .
\end{gathered}
$$

При любом $j$ задача (19)-(21) глобально разрешима, причем ее решение непрерывно по Гёльдеру (см. [26]-[28]). Таким образом, чтобы доказать теорему 1 необходимо получить не зависящую от $j$ оценку (6) для решения $u_{j}$. Продолжив $u_{j}(x, t)$ нулем вне $B_{j} \times(0, \infty)$, мы тем самым определим $u_{j}(x, t)$ в $\mathbb{R}^{N} \times(0, \infty)$. Все дальнейшие рассуждения будут проводиться для задачи (19)-(21) при произвольном фиксированном $j$, поэтому для удобства будем обозначать $u_{j}, u_{0 j}, B_{j}$ через $u, u_{0}, B$ соответственно.

Из результатов работы [12] следует справедливость следующей леммы.

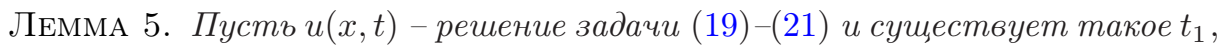
что для любых $t \in\left[0, t_{1}\right), x \in B_{2 R}\left(x_{0}\right)$ выполнено неравенство

$$
\frac{|x|^{l}}{(2 R)^{\lambda+1}} t u^{m+\lambda-2}(x, t)+t u^{p-1}(x, t) \leqslant 1 .
$$

Тогда для любых $t \in\left(0, t_{1}\right), \omega \geqslant 1$

$$
\|u(x, t)\|_{\infty, B_{R}\left(x_{0}\right)} \leqslant \gamma t^{-\frac{N-l}{H_{l, \omega}}}\left(\sup _{0<\tau<t} \int_{B_{2 R}\left(x_{0}\right)} \rho u^{\omega}(x, \tau) d x\right)^{\frac{\lambda+1-l}{H_{l, \omega}}} .
$$

Обозначим через $r_{\theta}(b, t)$ функцию, заданную неявно уравнением

$$
\frac{r_{\theta}^{N}}{\left(r_{\theta}+b\right)^{\alpha \theta+l}}=\Gamma^{N-\alpha \theta-l} t^{\frac{N-\alpha \theta-l}{G_{\alpha}}}+1,
$$

где $b \geqslant 0, \Gamma=C_{*}\left[u_{0}\right]_{\theta}^{\frac{m+\lambda-2}{G_{\alpha}}}, C_{*}$ - достаточно большое число, зависящее от параметров задачи, которое будет выбрано далее.

Очевидно, что функция $r_{\theta}(b, t)$ определена и непрерывна при $b \geqslant 0, t \geqslant 0$, кроме того, $r_{\theta}(b, 0)$ совпадает с функцией $r_{\theta}(b)$, определяемой уравнением (8). 
Также легко заметить, что $r_{\theta}(b, t)$ возрастает по каждому из своих аргументов и для любых $x_{0} \in \mathbb{R}^{N}, R \in\left[0,2 r_{\theta}\left(\left|x_{0}\right|, t\right)\right]$

$$
\frac{R^{N}}{\left(R+\left|x_{0}\right|\right)^{\alpha \theta+l}} \leqslant \gamma\left(\Gamma^{N-\alpha \theta-l} t^{\frac{N-\alpha \theta-l}{G_{\alpha}}}+1\right) .
$$

Введем обозначение

$$
\langle u\rangle_{\theta, t}=\sup _{0<\tau<t} \sup _{x_{0} \in \mathbb{R}^{N}} \sup _{R>r_{\theta}\left(\left|x_{0}\right|, \tau\right)}\left(2 R+\left|x_{0}\right|\right)^{\alpha}\left(f_{B_{R}\left(x_{0}\right)} \rho u^{\theta}(x, \tau) d x\right)^{\frac{1}{\theta}} .
$$

Тогда для любых $x_{0} \in \mathbb{R}^{N}, R \geqslant r_{\theta}\left(\left|x_{0}\right|, t\right)$

$$
\sup _{0<\tau<t} \int_{B_{2 R}\left(x_{0}\right)} \rho u^{\theta}(x, \tau) d x \leqslant \gamma \frac{R^{N}}{\left(R+\left|x_{0}\right|\right)^{\alpha \theta+l}}\langle u\rangle_{\theta, t}^{\theta} .
$$

Пусть

$$
\begin{gathered}
\Omega(t) \equiv \sup _{0<\tau<t} \sup _{x_{0} \in \mathbb{R}^{N}}\left\{\frac{\tau\left(r_{\theta}\left(\left|x_{0}\right|, \tau\right)+\left|x_{0}\right|\right)^{l}}{r_{\theta}^{\lambda+1}\left(\left|x_{0}\right|, \tau\right)}\|u(\cdot, \tau)\|_{\infty, \mathbb{R}^{N}}^{m+\lambda-2}\right\} \\
+\sup _{0<\tau<t}\left\{\tau\|u(\cdot, \tau)\|_{\infty, \mathbb{R}^{N}}^{p-1}\right\}, \\
T=\sup \{t: \Omega(t) \leqslant 1\} .
\end{gathered}
$$

Из непрерывности решения $u(x, t)$ следует, что $T>0$. Из леммы 5 и соотношений (9), (26) получаем следующую лемму.

Лемма 6. Пусть и $(x, t)-$ решение задачи (19)-(21). Тогда для любого $t \in$ $(0, T)$ u $q \geqslant 1$ справедливы оченки

$$
\begin{gathered}
\|u(\cdot, t)\|_{\infty, \mathbb{R}^{N}} \leqslant \gamma t^{-\frac{N-l}{H_{l, \theta}}}\left(\Gamma^{N-\alpha \theta-l} t^{\frac{N-\alpha \theta-l}{G \alpha}}+1\right)^{\frac{\lambda+1-l}{H_{l, \theta}}}\langle u\rangle_{\theta, t}^{\frac{(\lambda+1-l) \theta}{H_{l, \theta}}}, \\
\|u(\cdot, t)\|_{\infty, \mathbb{R}^{N}} \leqslant \gamma t^{-\frac{N-l}{H_{l, q}}}\left(\sup _{0<\tau<t} \int_{\mathbb{R}^{N}} \rho u^{q}(x, \tau) d x\right)^{\frac{\lambda+1-l}{H_{l, q}}} .
\end{gathered}
$$

ЗАмЕчаниЕ 5. Функция $u=u_{j}$ продолжена нулем вне $B_{j} \times(0, T)$, поэтому интеграл в правой части (29) конечен.

Лемма 7. Пусть $u(x, \tau)$ - решение задачи (19)-(21) и $\theta=1$. Тогда для любых $t \in(0, T), x_{0} \in \mathbb{R}^{N}, R \geqslant r_{1}\left(\left|x_{0}\right|, t\right)$

$$
\begin{aligned}
I \equiv \frac{1}{R} \int_{0}^{t} \int_{B_{2 R}\left(x_{0}\right)} & u^{m-1}|D u|^{\lambda} \zeta^{\lambda}(x) d x d \tau \leqslant \gamma \frac{\left(R+\left|x_{0}\right|\right)^{\frac{l}{\lambda+1}}}{R} \\
& \times \frac{R^{N}}{\left(R+\left|x_{0}\right|\right)^{\alpha+l}} t^{\frac{\lambda+1-l}{(\lambda+1) K_{l}}}\left(\Gamma^{N-\alpha-l} t^{\frac{N-\alpha-l}{G \alpha}}+1\right)^{\frac{(m+\lambda-2)(\lambda+1-l)}{(\lambda+1) K_{l}}} \\
& \times\langle u\rangle_{1, t}^{1+\frac{(\lambda+1-l)(m+\lambda-2)}{(\lambda+1) K_{l}}}
\end{aligned}
$$

где $K_{l}=H_{l, 1}$ и $\zeta(x)$ - дифферениируемая функиия такая, что $\zeta(x)=1$ при $x \in B_{R}\left(x_{0}\right), \zeta(x)=0$ nрu $x \notin B_{2 R}\left(x_{0}\right) u|D \zeta| \leqslant 1 / R$. 
ДоказАтеЛьСтво. Пусть $\varkappa=(2-m) / \lambda$ и $\beta \in\left((N-l)(1-\varkappa) / K_{l}, 1 / \lambda\right)$. Тогда

$$
I=\frac{1}{R} \int_{0}^{t} \int_{B_{2 R}\left(x_{0}\right)} u^{m-1}|D u|^{\lambda} \zeta^{\lambda} \tau^{\beta \frac{\lambda}{\lambda+1}} \tau^{-\beta \frac{\lambda}{\lambda+1}} u^{\varkappa \frac{\lambda}{\lambda+1}} u^{-\varkappa \frac{\lambda}{\lambda+1}} d x d \tau
$$

Применяя неравенство Гёльдера с показателями $(\lambda+1) / \lambda$ и $\lambda+1$, получаем

$$
\begin{aligned}
& I \leqslant \frac{\left(R+\left|x_{0}\right|\right)^{\frac{l}{\lambda+1}}}{R}\left(\int_{0}^{t} \int_{B_{2 R}\left(x_{0}\right)} \tau^{\beta} u^{m-1-\varkappa}|D u|^{\lambda+1} \zeta^{\lambda+1} d x d \tau\right)^{\frac{\lambda}{\lambda+1}} \\
& \quad \times\left(\int_{0}^{t} \int_{B_{2 R}\left(x_{0}\right)} \rho \tau^{-\beta \lambda} u^{m+\varkappa \lambda-1} d x d \tau\right)^{\frac{1}{\lambda+1}}=\frac{\left(R+\left|x_{0}\right|\right)^{\frac{l}{\lambda+1}}}{R} I_{1}^{\frac{\lambda}{\lambda+1}} I_{2}^{\frac{1}{\lambda+1}} .
\end{aligned}
$$

Для того чтобы оценить $I_{1}$, умножим уравнение (19) на $\tau^{\beta} u^{1-\varkappa} \zeta^{\lambda+1}$ и проинтегрируем по $B_{2 R}\left(x_{0}\right) \times(0, t)$ :

$$
\begin{aligned}
& \frac{1}{2-\varkappa} \int_{B_{2 R}\left(x_{0}\right)} \rho u^{2-\varkappa}(x, t) t^{\beta} \zeta^{\lambda+1}(x) d x-\frac{\beta}{2-\varkappa} \int_{0}^{t} \int_{B_{2 R}\left(x_{0}\right)} \rho u^{2-\varkappa} \tau^{\beta-1} \zeta^{\lambda+1} d x d \tau \\
&=-(1-\varkappa) I_{1}-(\lambda+1) \int_{0}^{t} \int_{B_{2 R}\left(x_{0}\right)} u^{m-\varkappa} \tau^{\beta}|D u|^{\lambda-1} D u D \zeta \zeta^{\lambda} d x d \tau \\
&+\int_{0}^{t} \int_{B_{2 R}\left(x_{0}\right)} \rho \tau^{\beta} u^{p+1-\varkappa} \zeta^{\lambda+1} d x d \tau .
\end{aligned}
$$

Применяя неравенство Юнга ко второму члену правой части, получим

$$
\begin{aligned}
I_{1} \leqslant \gamma_{1} \int_{0}^{t} \int_{B_{2 R}\left(x_{0}\right)} \rho u^{2-\varkappa} \tau^{\beta-1} d x d \tau \\
+\gamma_{1} \frac{\left(R+\left|x_{0}\right|\right)^{l}}{R^{\lambda+1}} \int_{0}^{t} \int_{B_{2 R}\left(x_{0}\right)} \rho u^{m+\lambda-\varkappa} \tau^{\beta} d x d \tau \\
\quad+\gamma_{1} \int_{0}^{t} \int_{B_{2 R}\left(x_{0}\right)} \rho \tau^{\beta} u^{p+1-\varkappa} d x d \tau=I_{3}+I_{4}+I_{5} .
\end{aligned}
$$

Из того, что $R \geqslant r_{1}\left(\left|x_{0}\right|, t\right)$ и $t \in(0, T)$, следует неравенство

$$
I_{4}+I_{5} \leqslant \gamma_{2} I_{3}
$$

Таким образом, чтобы оценить $I_{1}$, необходима оценка для $I_{3}$. Используя оценку (28), получаем

$$
\begin{aligned}
I_{3} \leqslant \int_{0}^{t} \tau^{\beta-1}\|u(\cdot, \tau)\|_{\infty, B_{2 R}\left(x_{0}\right)}^{1-\varkappa} \int_{B_{2 R}\left(x_{0}\right)} \rho u(x, \tau) d x d \tau \\
\leqslant \gamma_{4} \int_{0}^{t} \tau^{\beta-1} \tau^{-\frac{(N-l)(1-\varkappa)}{K_{l}}}\left[\Gamma^{N-\alpha-l} \tau^{\frac{N-\alpha-l}{G_{\alpha}}}+1\right]^{\frac{(1-\varkappa)(\lambda+1-l)}{K_{l}}} d \tau \\
\quad \times\langle u\rangle_{1, t}^{\frac{(\lambda+1-l)(1-\varkappa)}{K_{l}}} \sup _{0<\tau<t} \int_{B_{2 R}\left(x_{0}\right)} \rho u(x, \tau) d x .
\end{aligned}
$$


Учитывая выбор параметров $\beta, \varkappa$ и используя (26), приходим к оценкам

$$
\begin{gathered}
I_{3} \leqslant \gamma_{5} \frac{R^{N}}{\left(R+\left|x_{0}\right|\right)^{\alpha+l}} t^{\beta-\frac{(N-l)(1-\varkappa)}{k_{l}}}\left[\Gamma^{N-\alpha} t^{\frac{N-\alpha-l}{G_{\alpha}}}+1\right]^{\frac{(1-\varkappa)(\lambda+1-l)}{K_{l}}}\langle u\rangle_{1, t}^{1+\frac{(\lambda+1-l)(1-\varkappa)}{K_{l}}} \\
I_{2} \leqslant \gamma_{6} \int_{0}^{t} \tau^{-\beta \lambda} \int_{B_{2 R}\left(x_{0}\right)} \rho u d x d \tau \leqslant t^{1-\beta \lambda} \frac{R^{N}}{\left(R+\left|x_{0}\right|\right)^{\alpha+l}}\langle u\rangle_{1, t} .
\end{gathered}
$$

Из (31)-(35) следует справедливость леммы.

Отметим, что для любых $x_{0} \in \mathbb{R}^{N}, t \geqslant 0$ из того, что $R \geqslant r_{\theta}\left(\left|x_{0}\right|, t\right)$, следует

$$
\frac{\left(R+\left|x_{0}\right|\right)^{l}}{R^{\lambda+1}} \leqslant\left(\frac{R}{R+\left|x_{0}\right|}\right)^{\frac{(\lambda+1)(\alpha \theta+l)-l N}{N-\alpha \theta-l}}\left[\Gamma^{N-\alpha \theta-l} t^{\frac{N-\alpha \theta-l}{G_{\alpha}}}+1\right]^{-\frac{\lambda+1-l}{N-\alpha \theta-l}},
$$

а поскольку значение $\theta$ выбрано так, чтобы

$$
\frac{(\lambda+1)(\alpha \theta+l)-l N}{N-\alpha \theta-l} \geqslant 0,
$$

имеем

$$
\frac{\left(R+\left|x_{0}\right|\right)^{l}}{R^{\lambda+1}} \leqslant\left[\Gamma^{N-\alpha \theta-l} t^{\frac{N-\alpha \theta-l}{G_{\alpha}}}+1\right]^{-\frac{\lambda+1-l}{N-\alpha \theta-l}} .
$$

Лемма 8. Пусть $и(x, t)$ - решение задачи (19)-(21). Тогда для любых $t \in$ $[0, T)$ имеет место оценка

$$
\begin{aligned}
\langle u\rangle_{\theta, t}^{\theta} \leqslant \widetilde{C}\left[u_{0}\right]_{\theta}^{\theta}+\gamma C_{*}^{-\varkappa(\theta) \frac{G_{\alpha}(\lambda+1-l) \theta}{H_{l, \theta}}}\left[\frac{\langle u\rangle_{\theta, t}}{\left[u_{0}\right]_{\theta}}\right]^{\frac{(m+\lambda-2)(\lambda+1-l) \theta}{H_{l, \theta}} \varkappa(\theta)}\langle u\rangle_{\theta, t}^{\theta} \\
+\gamma \int_{0}^{t}\|u(\cdot, \tau)\|_{\infty, \mathbb{R}^{N}}^{p-1} d \tau\langle u\rangle_{\theta, t}^{\theta},
\end{aligned}
$$

где постоянная $\widetilde{C}$ зависит только лишь от параметров задачи, а $\varkappa(\theta)=1$ при $\theta>1$ и $\varkappa(\theta)=1 /(\lambda+1)$ при $\theta=1$.

ДоказАтельство. Рассмотрим сначала случай $\theta=1$. Пусть $R \geqslant r_{1}\left(\left|x_{0}\right|, t\right)$ и $\zeta(x)$ - функция из условия леммы 7. Умножим уравнение $(19)$ на $\zeta^{\lambda+1}(x)$ и проинтегрируем по $B_{2 R}\left(x_{0}\right) \times(0, t)$ при $t \in(0, T)$

$$
\begin{array}{r}
\int_{B_{R}\left(x_{0}\right)} \rho u(x, t) d x \leqslant \int_{B_{2 R}\left(x_{0}\right)} \rho u_{0}(x) d x+\frac{\gamma}{R} \int_{0}^{t} \int_{B_{2 R}\left(x_{0}\right)} u^{m-1}|D u|^{\lambda} \zeta^{\lambda} d x d \tau \\
+\int_{0}^{t} \int_{B_{2 R}\left(x_{0}\right)} \rho u^{p} d x d \tau .
\end{array}
$$

Умножим полученное неравенство на $\left(2 R+\left|x_{0}\right|\right)^{\alpha} / \int_{B_{2 R}\left(x_{0}\right)} \rho d x$ и применим $(30)$. Тогда

$$
\left(R+\left|x_{0}\right|\right)^{\alpha} f_{B_{R}\left(x_{0}\right)} \rho u(x, t) d x \leqslant \widetilde{C}\left(2 R+\left|x_{0}\right|\right)^{\alpha} f_{B_{2 R}\left(x_{0}\right)} \rho u_{0} d x+\gamma\left(\frac{\left(R+\left|x_{0}\right|\right)^{l}}{R^{\lambda+1}}\right)^{\frac{1}{\lambda+1}}
$$




$$
\begin{gathered}
\times t^{\frac{\lambda+1-l}{(\lambda+1) K_{l}}\left[\Gamma^{N-\alpha-l} t^{\frac{N-\alpha-l}{G_{\alpha}}}+1\right]^{\frac{(m+\lambda-2)(\lambda+1-l)}{(\lambda+1) K_{l}}}\langle u\rangle_{1, t}^{1+\frac{(\lambda+1-l)(m+\lambda-2)}{(\lambda+1) K_{l}}}} \\
\quad+\gamma \int_{0}^{t}\|u(\cdot, \tau)\|_{\infty, \mathbb{R}^{N}}^{p-1} d \tau \sup _{0<\tau<t}\left(2 R+\left|x_{0}\right|\right)^{\alpha} f_{B_{2 R}\left(x_{0}\right)} \rho u d x .
\end{gathered}
$$

Используя (36) во втором слагаемом справа, получаем (37) при $\theta=1$.

Теперь рассмотрим случай $\theta>1$. Пусть $R \geqslant r_{\theta}\left(\left|x_{0}\right|, t\right)$. Умножим уравнение (19) на $u^{\theta-1} \zeta^{\lambda+1}$ и проинтегрируем по $B_{2 R}\left(x_{0}\right) \times(0, t)$ при $t \in(0, T)$ :

$$
\begin{aligned}
\frac{1}{\theta} \int_{B_{2 R}\left(x_{0}\right)} \rho u^{\theta}(x, t) d x & -\frac{1}{\theta} \int_{B_{2 R}\left(x_{0}\right)} \rho u_{0}^{\theta}(x, t) d x \\
\leqslant-(\theta-1) \int_{0}^{t} & \int_{B_{2 R}\left(x_{0}\right)} u^{m-1}|D u|^{\lambda+1} u^{\theta-2} \zeta^{\lambda+1} d x d \tau \\
& +\frac{\lambda+1}{R} \int_{0}^{t} \int_{B_{2 R}\left(x_{0}\right)} u^{m+\theta-2}|D u|^{\lambda} \zeta^{\lambda} d x d \tau \\
& +\int_{0}^{t} \int_{B_{2 R}\left(x_{0}\right)} \rho u^{p} u^{\theta-1} \zeta^{\lambda+1} d x d \tau=-I_{1}+I_{2}+I_{3} .
\end{aligned}
$$

Оценим $I_{2}$ с помощью неравенства Юнга:

$$
\begin{aligned}
I_{2} & \leqslant \varepsilon I_{1}+\frac{\gamma(\varepsilon)}{R^{\lambda+1}} \int_{0}^{t} \int_{B_{2 R}\left(x_{0}\right)} u^{m+\theta+\lambda-2} d x d \tau \\
& \leqslant \varepsilon I_{1}+\gamma(\varepsilon) \frac{\left(R+\left|x_{0}\right|\right)^{l}}{R^{\lambda+1}} \int_{0}^{t}\|u(\cdot, \tau)\|_{\infty, \mathbb{R}^{N}}^{m+\lambda-2} d \tau \sup _{0<\tau<t} \int_{B_{2 R}\left(x_{0}\right)} \rho u^{\theta} d x .
\end{aligned}
$$

Используя соотношения (36) и (28), из (38) и (39) получаем

$$
\begin{aligned}
& \int_{B_{R}\left(x_{0}\right)} \rho u^{\theta}(x, t) d x \leqslant \int_{B_{2 R}\left(x_{0}\right)} \rho u_{0}^{\theta}(x) d x+\gamma t^{1-\frac{(N-l)(m+\lambda-2)}{H_{l, \theta}}} \\
& \quad \times\left[\Gamma^{N-\alpha \theta-l} t^{\frac{N-\alpha \theta-l}{G_{\alpha}}}+1\right]^{-\frac{\lambda+1-l}{N-\alpha \theta-l}+\frac{(\lambda+1-l)(m+\lambda-2)}{H_{l, \theta}}}\langle u\rangle_{\theta, t}^{\frac{(\lambda+1-l)(m+\lambda-2) \theta}{H_{l, \theta}}} \\
& \quad \times \sup _{0<\tau<t} \int_{B_{2 R}\left(x_{0}\right)} \rho u^{\theta} d x+\gamma \int_{0}^{t}\|u(\cdot, \tau)\|_{\infty, \mathbb{R}^{N}}^{p-1} d \tau \sup _{0<\tau<t} \int_{B_{2 R}\left(x_{0}\right)} \rho u^{\theta} d x .
\end{aligned}
$$

Умножая обе части этого неравенства на $\left(2 R+\left|x_{0}\right|\right)^{\alpha} / \int_{B_{2}\left(x_{0}\right)} \rho d x$, получаем (37) при $\theta>1$. Лемма доказана.

Введем следующие обозначения:

$$
\begin{aligned}
\mu(t) & =\sup _{0<\tau<t} \int_{\mathbb{R}^{N}} \rho u^{q}(x, \tau) d x, & \mu_{0} & =\int_{\mathbb{R}^{N}} \rho u_{0}^{q}(x) d x, \\
T_{\mu} & =\sup \left\{t: \mu(t) \leqslant 2 \mu_{0}\right\}, & T_{\langle\rangle} & =\sup \left\{t:\langle u\rangle_{t, \theta}^{\theta} \leqslant 2 \widetilde{C}\left[u_{0}\right]_{\theta}^{\theta}\right\},
\end{aligned}
$$

где $\widetilde{C}-$ постоянная из условия леммы 8 .

Лемма 9. Пусть $q>Q$ и выполнено (5) с достаточно мальм $\delta$. Тогда

$$
\min \left\{T_{\mu}, T\right\}>1 \text {. }
$$


ДокАЗАТЕЛЬСтво. Рассмотрим два случая:

1) $T_{\mu}<T$

2) $T \leqslant T_{\mu}$.

В случае 1$)$ предположим, что $T_{\mu} \leqslant 1$; тогда, умножая уравнение (19) на $u^{q-1}$ и интегрируя по $\mathbb{R}^{N} \times\left(0, T_{\mu}\right)$, получим

$$
\mu\left(T_{\mu}\right) \leqslant \mu_{0}+\gamma \mu\left(T_{\mu}\right) \int_{0}^{T_{\mu}}\|u(\cdot, \tau)\|_{\infty, \mathbb{R}^{N}}^{p-1} d \tau .
$$

Используя оценку (29) и учитывая, что $q>Q$, приходим к неравенству

$$
\begin{aligned}
\int_{0}^{T_{\mu}}\|u(\cdot, \tau)\|_{\infty, \mathbb{R}^{N}}^{p-1} d \tau & \leqslant \gamma_{1} T_{\mu}^{1-\frac{(p-1)(N-l)}{H_{l, q}}} \mu\left(T_{\mu}\right)^{\frac{(p-1)(\lambda+1-l)}{H_{l, q}}} \\
& \leqslant \gamma_{2} T_{\mu}^{1-\frac{(p-1)(N-l)}{H_{l, q}}} \mu_{0}^{\frac{(p-1)(\lambda+1-l)}{H_{l, q}}}
\end{aligned}
$$

Из (40), (41) при достаточно малом $\delta$ получаем

$$
\mu\left(T_{\mu}\right) \leqslant \mu_{0}+\gamma_{3} \delta^{\frac{(p-1)(\lambda+1-l)}{H_{l, q}}} \mu\left(T_{\mu}\right) \leqslant \mu_{0}+\frac{1}{3} \mu\left(T_{\mu}\right) .
$$

Таким образом,

$$
\mu\left(T_{\mu}\right) \leqslant \frac{3}{2} \mu_{0}
$$

что противоречит определению $T_{\mu}$. Значит, $T_{\mu}>1$.

В случае 2$)$, используя оценку (29) и неравенство (36), получим

$$
\begin{aligned}
\Omega(t) \leqslant \gamma & \sup _{0<\tau<T}\left\{\tau^{1-\frac{(p-1)(N-l)}{H_{l, q}}} \mu(\tau)^{\frac{(\lambda+1-l)(m+\lambda-2)}{H_{l, q}}}\right\} \\
& +\gamma \sup _{0<\tau<T}\left\{\tau^{1-\frac{(p-1)(N-l)}{H_{l, q}}} \mu(\tau)^{\frac{(p-1)(\lambda+1-l)}{H_{l, q}}}\right\} .
\end{aligned}
$$

Поскольку $q>Q$, при достаточно малом $\delta$ приходим к неравенству

$$
\Omega(T) \leqslant \gamma_{1} \mu_{0}^{\frac{(\lambda+1-l)(m+\lambda-2)}{H_{l, q}}}+\gamma_{2} \mu_{0}^{\frac{(p-1)(\lambda+1-l)}{H_{l, q}}}<\frac{1}{2},
$$

что противоречит определению $T$, если $T<\infty$. Значит, $T>1$. Лемма доказана.

Лемма 10. Пусть $q>Q, p>p_{\alpha}^{*}(l)$ и выполнено (5) с достаточно малым $\delta$. Тогда $T_{\langle\rangle} \geqslant T$.

ДокАЗАТЕЛЬСтво. Предположим, что $T_{\langle\rangle}<T$; тогда

$$
\frac{\langle u\rangle_{\theta, T_{\langle\rangle}}^{\theta}}{\left[u_{0}\right]_{\theta}^{\theta}} \leqslant 2 \widetilde{C}
$$

и, выбирая $C_{*}$ достаточно большим, из (37) получим

$$
\langle u\rangle_{\theta, T_{\langle\rangle}}^{\theta} \leqslant \widetilde{C}\left[u_{0}\right]_{\theta}^{\theta}+\frac{1}{6} \widetilde{C}\langle u\rangle_{\theta, T_{\langle\rangle}}^{\theta}+\gamma \int_{0}^{T_{\langle\rangle}}\|u(\cdot, \tau)\|_{\infty, \mathbb{R}^{N}}^{p-1} d \tau\langle u\rangle_{\theta, T_{\langle\rangle}}^{\theta} .
$$


Если $T_{\langle\rangle} \leqslant 1$, то из условия $q>Q$ и оценки $(29)$ имеем

$$
\int_{0}^{T_{\langle\rangle}}\|u(\cdot, \tau)\|_{\infty, \mathbb{R}^{N}}^{p-1} d \tau \leqslant \gamma_{1} \int_{0}^{1} \tau^{-\frac{(N-l)(p-1)}{H_{l, q}}} d \tau \mu(1)^{\frac{(p-1)(\lambda+1-l)}{H_{l, q}}} \leqslant \gamma_{2} \delta^{\frac{(p-1)(\lambda+1-l)}{H_{l, q}}} .
$$

Если $T_{\langle\rangle}>1$, то

$$
\begin{aligned}
\int_{0}^{T_{\langle 〉}}\|u(\cdot, \tau)\|_{\infty, \mathbb{R}^{N}}^{p-1} d \tau & =\int_{0}^{1}\|u(\cdot, \tau)\|_{\infty, \mathbb{R}^{N}}^{p-1} d \tau+\int_{1}^{T_{\langle 〉}}\|u(\cdot, \tau)\|_{\infty, \mathbb{R}^{N}}^{p-1} d \tau \\
& \leqslant \gamma_{3} \delta^{\frac{(p-1)(\lambda+1-l)}{H_{l, q}}}+\int_{1}^{T_{\langle\rangle}}\|u(\cdot, \tau)\|_{\infty, \mathbb{R}^{N}}^{p-1} d \tau .
\end{aligned}
$$

При $p>p_{\alpha}^{*}(l)$ из оценки (28) следует

$$
\begin{aligned}
\int_{1}^{T_{\langle\rangle}} \| u( & , \tau) \|_{\infty, \mathbb{R}^{N}}^{p-1} d \tau \leqslant \gamma_{4} \int_{1}^{T_{\langle\rangle}} \tau^{-\frac{(N-l)(p-1)}{H_{l, \theta}}} \\
& \times \max \left\{\Gamma^{\frac{(N-\alpha \theta-l)(\lambda+1-l)}{H_{l, \theta}}} \tau^{\frac{(N-\alpha \theta-l)(\lambda+1-l)}{G_{\alpha} H_{l, \theta}}}, 1\right\}^{p-1} d \tau\langle u\rangle_{\theta, T_{\langle\rangle}}^{\frac{(\lambda+1-l) \theta(p-1)}{H_{l, \theta}}} \\
\leqslant & \gamma_{5} \int_{1}^{T_{\langle\rangle}} \tau^{-\frac{\alpha(p-1)}{G_{\alpha}}} \max \left\{1, \tau^{-\frac{(p-1)(\lambda+1-l)(N-l-\theta \alpha)}{H_{l, \theta} G_{\alpha}}}\right\} d \tau \\
& \times \max \left\{\Gamma^{\frac{(N-\alpha \theta-l)(\lambda+1-l)(p-1)}{H_{l, \theta}}}, 1\right\}\left[u_{0}\right]_{\theta}^{\frac{(\lambda+1-l) \theta(p-1)}{H_{l, \theta}}} \\
\leqslant & \gamma_{6} \max \left\{\left(C_{*} \delta^{\frac{m+\lambda-2}{G_{\alpha}}}\right)^{\frac{(N-\alpha \theta-l)(\lambda+1-l)(p-1)}{H_{l, \theta}}}, 1\right\} \delta^{\frac{(\lambda+1-l) \theta(p-1)}{H_{l, \theta}}} .
\end{aligned}
$$

Выбирая $\delta$ достаточно малым, из (43)-(46) получаем

$$
\langle u\rangle_{\theta, T_{\langle\rangle}}^{\theta} \leqslant \widetilde{C}\left[u_{0}\right]_{\theta}^{\theta}+\frac{1}{6} \widetilde{C}\langle u\rangle_{\theta, T_{\zeta\rangle}}^{\theta}+\frac{1}{6} \widetilde{C}\langle u\rangle_{\theta, T_{\langle\rangle}}^{\theta},
$$

то есть

$$
\langle u\rangle_{\theta, T_{\langle\rangle}}^{\theta} \leqslant \frac{3}{2} \widetilde{C}\left[u_{0}\right]_{\theta}^{\theta},
$$

что противоречит определению $T_{\langle\rangle}$, значит, $T_{\langle\rangle} \geqslant T$. Лемма доказана.

Для завершения доказательства теоремы 1 остается показать, что $T=\infty$ при $p>p_{\alpha}^{*}(l)$. Предположим, что $p>p_{\alpha}^{*}(l)$. Тогда, используя $(28)$ и $(36)$ для первого слагаемого $\Omega(t)$ и $(28),(29)$ для второго слагаемого, получим

$$
\begin{aligned}
\Omega(T) \leqslant & \gamma \sup _{0<\tau<T}\left\{\tau^{\frac{\theta(\lambda+1-l)}{H_{l, \theta}}}\left(\Gamma^{N-\alpha \theta-l} \tau^{\frac{N-\alpha \theta}{G_{\alpha}}}+1\right)^{-\frac{(\lambda+1-l) \theta G_{\alpha}}{(N-\alpha \theta-l) H_{l, \theta}}}\right\}\left[u_{0}\right]_{\theta}^{\frac{(\lambda+1-l) \theta(m+\lambda-2)}{H_{l, \theta}}} \\
& +\gamma \sup _{0<\tau<1}\left\{\tau^{1-\frac{(N-l)(p-1)}{H_{l, q}}}\right\} \mu_{0}^{\frac{(\lambda+1-l)(p-1)}{H_{l, q}}} \\
& +\gamma \sup _{1<\tau<T}\left\{\tau^{1-\frac{(N-l)(p-1)}{H_{l, \theta}}}\left(\Gamma^{N-\alpha \theta-l} \tau^{\frac{N-\alpha \theta-l}{G_{\alpha}}}+1\right)^{\frac{(\lambda+1-l)(p-1)}{H_{l, \theta}}}\right\}\left[u_{0}\right]^{\frac{(\lambda+1-l)(p-1) \theta}{H_{l, \theta}}} \\
\leqslant & \gamma_{1} C_{*}^{-\frac{\theta(\lambda+1-l) G_{\alpha}}{H_{l, \theta}}}+\mu_{0}^{\frac{(\lambda+1-l)(p-1)}{H_{l, q}}}+\left[\Gamma^{N-\alpha \theta-l}+1\right]^{\frac{(\lambda+1-l)(p-1)}{H_{l, \theta}}}\left[u_{0}\right]_{\theta}^{\frac{(\lambda+1-l) \theta(p-1)}{H_{l, \theta}}} .
\end{aligned}
$$

Выбирая $C_{*}$ достаточно большим, а $\delta$ достаточно малым, получаем $\Omega(T) \leqslant 1 / 2$, что невозможно при конечном $T$. 


\section{§4. Доказательство теоремы 2}

Пусть $\zeta(x)$ - гладкая срезающая функция такая, что $\zeta(x)=1$ при $x \in$ $B_{R}\left(x_{0}\right), \zeta(x)=0$ при $x \notin B_{2 R}\left(x_{0}\right)$ и $|D \zeta|<\gamma R^{-1}$, и пусть $\varepsilon, s>0$. Тогда, умножив обе части уравнения (19) на $(u+\varepsilon)^{-\theta} \zeta^{s}$ и проинтегрировав по $B_{2 R}\left(x_{0}\right)$, получаем

$$
\begin{aligned}
\frac{d}{d t} \int_{B_{2 R}\left(x_{0}\right)} \rho(u(x, t)+\varepsilon)^{1-\theta} \zeta^{s} d x & \geqslant \gamma_{1} \int_{B_{2 R}\left(x_{0}\right)} u^{m-1}|D u|^{\lambda+1}(u+\varepsilon)^{-(1+\theta)} \zeta^{s} d x \\
& -\gamma_{2} \int_{B_{2 R}\left(x_{0}\right)} u^{m-1}|D u|^{\lambda}(u+\varepsilon)^{-\theta} \zeta^{s-1}|D \zeta| d x \\
& +\gamma_{3} \int_{B_{2 R}\left(x_{0}\right)} \rho u^{p}(u+\varepsilon)^{-\theta} \zeta^{s} d x .
\end{aligned}
$$

Применяя ко второму слагаемому правой части неравенство Юнга с достаточно малым $\nu$ и переходя к пределу при $\varepsilon \rightarrow 0$, получаем

$$
\begin{aligned}
& \frac{d}{d t} \int_{B_{2 R}\left(x_{0}\right)} \rho u^{1-\theta} \zeta^{s} d x \geqslant \gamma_{4}(\nu) \int_{B_{2 R}\left(x_{0}\right)} u^{m-\theta-2}|D u|^{\lambda+1} \zeta^{s} d x \\
& \quad-\frac{\gamma_{5}(\nu)}{R^{\lambda+1}} \int_{B_{2 R}\left(x_{0}\right)} u^{m+\lambda-\theta-1} \zeta^{s-\lambda-1} d x+\gamma_{3} \int_{B_{2 R}\left(x_{0}\right)} \rho u^{p-\theta} \zeta^{s} d x=I_{1}-I_{2}+I_{3} .
\end{aligned}
$$

Снова применяя неравенство Юнга с достаточно малым $\nu>0$ и выбирая

$$
s>\frac{(\lambda+1)(p-\theta)}{p-m-\lambda+1},
$$

получаем

$$
I_{2} \leqslant \nu I_{3}+\gamma_{6}(\nu) R^{-\frac{(\lambda+1)(p-\theta)}{p-m-\lambda+1}} \int_{B_{2 R}\left(x_{0}\right)} \rho^{-\frac{m+\lambda-\theta-1}{p-m-\lambda+1}} d x .
$$

Отсюда, используя (9), имеем

$$
I_{2} \leqslant \nu I_{3}+\gamma_{7}(\nu) F\left(R,\left|x_{0}\right|\right)
$$

где

$$
F\left(R,\left|x_{0}\right|\right)=R^{N-\frac{(\lambda+1)(p-\theta)}{p-m-\lambda+1}}\left(R+\left|x_{0}\right|\right)^{\frac{l(m+\lambda-\theta-1)}{p-m-\lambda+1}} .
$$

Обозначим $E=E(t)=\int_{B_{2 R}\left(x_{0}\right)} \rho u^{1-\theta}(x, t) d x$ и, учитывая малость $\nu$, из соотношений (47), (48) получим

$$
\frac{d}{d t} E \geqslant \gamma_{8} I_{3}-\gamma_{7} F\left(R,\left|x_{0}\right|\right)
$$

Применяя неравенство Гёльдера и неравенство (9), получим

$$
E \leqslant \gamma_{9} I_{3}^{\frac{1-\theta}{p-\theta}}\left(R^{N}\left(R+\left|x_{0}\right|\right)^{-l}\right)^{\frac{p-1}{p-\theta}},
$$

следовательно,

$$
I_{3} \geqslant \gamma_{10} E^{\frac{p-\theta}{1-\theta}} H\left(R,\left|x_{0}\right|\right),
$$

где $H\left(R,\left|x_{0}\right|\right)=\left(R^{N}\left(R+\left|x_{0}\right|\right)^{-l}\right)^{-(p-1) /(1-\theta)}$. 
Таким образом, из (49) получаем неравенство для $E$ :

$$
\frac{d}{d t} E \geqslant \gamma_{11} E^{\frac{p-\theta}{1-\theta}} H\left(R,\left|x_{0}\right|\right)-\gamma_{7} F\left(R,\left|x_{0}\right|\right) .
$$

Покажем, что существует положительное $R_{1}<\infty$ такое, что для любых $t \geqslant 0$, $R \geqslant R_{1}$ выполнено

$$
C E^{\frac{p-\theta}{1-\theta}} H\left(R,\left|x_{0}\right|\right) \geqslant F\left(R,\left|x_{0}\right|\right),
$$

где $C=$ const $>0$ - достаточно малое число.

Действительно, (51) равносильно неравенству

$$
\begin{aligned}
C\left(\left(R+\left|x_{0}\right|\right)^{\alpha(1-\theta)}\right. & \left.f_{B_{2 R}\left(x_{0}\right)} \rho u^{1-\theta}(x, t) d x\right)^{\frac{p-\theta}{1-\theta}} \\
& \geqslant \gamma_{12}\left(\frac{\left(R+\left|x_{0}\right|\right)^{\alpha(1-\theta)+l}}{R^{N}}\right)^{\frac{p-\theta}{1-\theta}} \frac{F\left(R,\left|x_{0}\right|\right)}{H\left(R,\left|x_{0}\right|\right)} \\
& =\gamma_{12}\left(R^{-\frac{\lambda+1}{p-m-\lambda+1}}\left(R+\left|x_{0}\right|\right)^{\frac{l}{p-m-\lambda+1}+\alpha}\right)^{p-\theta}
\end{aligned}
$$

правая часть которого убывает по $R$ при $p<p_{\alpha}^{*}(l)$. Значит, в силу условия (7) найдется такое достаточно большое $R_{1}>0$, что (51) выполнено при $t=0$ и $R \geqslant R_{1}$ со сколь угодно малым $C$. Однако тогда из $(50)$, (51) вытекает, что $E(t)$ - возрастающая функция и, следовательно, (51) выполнено при всех $t \geqslant 0, R \geqslant R_{1}$.

Таким образом, из (50), (51) следует

$$
\frac{d}{d t} E(t) \geqslant \gamma E^{\frac{p-\theta}{1-\theta}} H\left(R,\left|x_{0}\right|\right) .
$$

Интегрируя это неравенство по интервалу $(0, t)$, получим

$$
E(t) \geqslant \frac{E(0)}{\left[1-\gamma H\left(R,\left|x_{0}\right|\right) t\right]^{\frac{1-\theta}{p-1}}},
$$

откуда следует, что $E(t) \rightarrow \infty$ при $t \rightarrow T=1 /\left(\gamma H\left(R,\left|x_{0}\right|\right)\right)$.

\section{Список литературы}

1. S. Kaplan, "On the growth of solutions of quasi-linear parabolic equations", Comm. Pure Appl. Math., 16:3 (1963), 305-330.

2. H. Fujita, "On the blowing up of solutions of the Cauchy problem for $u_{t}=\Delta u+u^{1+\alpha}$ ", J. Fac. Sci. Univ. Tokyo Sect. I, 13 (1966), 109-124.

3. А. А. Самарский, В.А. Галактионов, С.П. Курдюмов, А.П. Михайлов, "О неограниченных решениях задачи Коши для параболического уравнения $u_{t}=$ $\nabla\left(u^{\sigma} \nabla u\right) u+u^{\beta}$, , Докл. АН СССР, 252:6 (1980), 1362-1364; англ. пер.: V.A. Galaktionov, S.P. Kurdyumov, A.P. Mikhajlov, A. A. Samarskij, "Unbounded solutions of the Cauchy problem for the parabolic equation $u_{t}=\nabla\left(u^{\sigma} \nabla u\right) u+u^{\beta}$ ", Soviet Phys. Dokl., 25 (1980), 458-459.

4. А. А. Самарский, В.А. Галактионов, С.П. Курдюмов, А.П. Михайлов, Режимы с обострением в задачах для квазилинейных параболических уравнений, Наука, M., 1987; англ. пер.: А. А. Samarskii, V. A. Galaktionov, S. P. Kurdyumov, A. P. Mikhailov, Blow-up in quasilinear parabolic equations, de Gruyter Exp. Math., 19, de Gruyter, Berlin, 1995. 
5. D. Andreucci, E. Di Benedetto, "On the Cauchy problem and initial traces for a class of evolution equations with strongly nonlinear sources", Ann. Scuola Norm. Sup. Pisa Cl. Sci. (4), 18:3 (1991), 363-441.

6. V.A. Galaktionov, "Blow-up for quasilinear heat equations with critical Fujita's exponents", Proc. Roy. Soc. Edinburgh Sect. A, 124:3 (1994), 517-525.

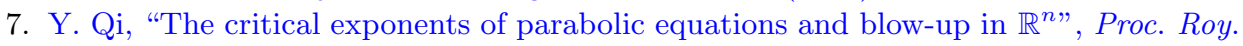
Soc. Edinburgh Sect. A, 128:1 (1998), 123-136.

8. V.A. Galaktionov, H. A. Levine, "A general approach to critical Fujita exponents in nonlinear parabolic problems", Nonlinear Anal., 34:7 (1998), 1005-1027.

9. D. Andreucci, A. F. Tedeev, "A Fujita type result for a degenerate Neumann problem in domains with noncompact boundary", J. Math. Anal. Appl., 231:2 (1999), 543-567.

10. H. A. Levine, "The role of critical exponents in blowup theorems", SIAM Rev., 32:2 (1990), 262-288.

11. K. Deng, H. A. Levine, "The role of critical exponents in blow-up theorems: the sequel", J. Math. Anal. Appl., 243:1 (2000), 85-126.

12. А. В. Мартыненко, А.Ф. Тедеев, "О поведении решений задачи Коши для вырождающегося параболического уравнения с неоднородной плотностью и источником", Ж. вычисл. матем. и матем. физ., 48:7 (2008), 1214-1229; англ. пер.: A. V. Martynenko, A. F. Tedeev, "On the behavior of solutions to the Cauchy problem for a degenerate parabolic equation with inhomogeneous density and a source", Comput. Math. Math. Phys., 48:7 (2008), 1145-1160.

13. P. Rosenau, S. Kamin, "Non-linear diffusion in a finite mass medium", Comm. Pure Appl. Math., 35:1 (1982), 113-127.

14. S. Kamin, P. Rosenau, "Propagation of thermal waves in an inhomogeneous medium", Comm. Pure Appl. Math., 34:6 (1981), 831-852.

15. S. Kamin, R. Kersner, "Disappearance of interfaces in finite time", Meccanica, 28:2 (1993), 117-120.

16. M. Guedda, D. Hilhorst, M. A. Peletier, "Disappearing interfaces in nonlinear diffusion", Adv. Math. Sci. Appl., 7:2 (1997), 695-710.

17. V.A. Galaktionov, J. R. King, "On the behaviour of blow-up interfaces for an inhomogeneous filtration equation", IMA J. Appl. Math., 57:1 (1996), 53-77.

18. R. Kersner, G. Reyes, A. Tesei, "On a class of parabolic equations with variable density and absorption", Adv. Differential Equations, 7:2 (2002), 155-176.

19. В. А. Галактионов, С. Камин, Р. Керснер, Дж. Л. Вакуэ, "Промежуточные асимптотики для неоднородной нелинейной теплопроводности", Тр. сем. им. И. Г. Петровского, Изд-во Моск. ун-та, М., 2003, 61-92; англ. пер.: V. A. Galaktionov, S. Kamin, R. Kersner, J. L. Vazquez, "Intermediate asymptotics for inhomogeneous nonlinear heat conduction", J. Math. Sci., 120:3 (2004), 1277-1294.

20. А.Ф. Тедеев, "Условия существования и несуществования в целом по времени компактного носителя решений задачи Коши для квазилинейных вырождающихся параболических уравнений", Сиб. матем. журн., 45:1 (2004), 189-200; англ. пер.: A.F. Tedeev, "Conditions for the time global existence and nonexistence of a compact support of solutions to the Cauchy problem for quasilinear degenerate parabolic equations", Siberian Math. J., 45:1 (2004), 155-164.

21. A.F. Tedeev, "The interface blow-up phenomenon and local estimates for doubly degenerate parabolic equations", Appl. Anal., 86:6 (2007), 755-782.

22. K. Mukai, K. Mochuzuki, Q. Huang, "Large time behavior and life span for a quasilinear parabolic equation with slowly decaying initial values", Nonlinear Anal., 39:1 (2000), 33-45.

23. Н. В. Афанасьева, А. Ф. Тедеев, “Теоремы типа Фуджиты для квазилинейных параболических уравнений в случае медленно стремящихся к нулю начальных данных", Матем. сб., 195:4 (2004), 3-22; англ. пер.: N. V. Afanasieva, A. F. Tedeev, 
"Fujita type theorems for quasilinear parabolic equations with initial data slowly decaying to zero", Sb. Math., 195:4 (2004), 459-478.

24. D. Andreucci, A.F. Tedeev, "Universal bounds at the blow-up time for nonlinear parabolic equations", Adv. Differential Equations, 10:1 (2005), 89-120.

25. D. Andreucci, "Degenerate parabolic equations with initial data measures", Trans. Amer. Math. Soc., 340:10 (1997), 3911-3923.

26. F. Bernis, "Existence results for doubly nonlinear higher order parabolic equations on unbounded domains", Math. Ann., 279:3 (1988), 373-394.

27. H. W. Alt, S. Luckhaus, "Quasilinear elliptic-parabolic differential equations", Math. Z., 183:3 (1983), 311-341.

28. А. В. Мартыненко, А.Ф. Тедеев, "Регулярность решений вырождающихся параболических уравнений с неоднородной плотностью”, Укр. матем. вестн., 5:1 (2008), 116-145; англ. пер.: А. V. Martynenko, A.F. Tedeev, "Regularity of solutions of degenerate parabolic equations with nonhomogeneous density", Ukr. Math. Bull., 5:1 (2008), 117-145.

A. В. Maptыhehko (А. V. Martynenko)

Луганский национальный университет

им. Т. Шевченко, Украина

А. Ф. Тедеев (А. F. Tedeev)

Институт прикладной математики и механики

НАН Украины, г. Донецк

E-mail: tedeev@iamm.ac.donetsk.ua

B. H. Шраmehko (V. N. Shramenko)

Национальный технический университет Украины

"Киевский политехнический институт"
Поступило в редакцию

31.01 .2011 